\title{
Outage Capacity of Two-Phase Space-Time Coded Cooperative Multicasting
}

\author{
Aitor del Coso ${ }^{\dagger}$, Osvaldo Simeone ${ }^{\S}$, Yeheskel Bar-ness ${ }^{\S}$ and Christian Ibars ${ }^{\dagger}$ \\ $\dagger$ Centre Tecnològic de Telecomunicacions de Catalunya (CTTC) \\ Parc Mediterrani de la Tecnologia - 08860 - Castelldefels (Spain) \\ $\S$ CCSPR, New Jersey Institute of Technology. \\ University Heights - 07102 - Newark, NJ \\ email: \{adelcoso,cibars\}@cttc.es, \{osvaldo.simeone,yeheskel.barness\}@njit.edu
}

\begin{abstract}
Multi-path fading poses severe limitations to the reliable transmission rate of multicast in wireless networks. To improve robustness and throughput, cooperation among the nodes of the network can be used to crate spatial diversity and thus overcome fading impairments. This paper proposes a two-phase, space-time coded, cooperative multicast protocol, and analyzes its capacity performance in finite size networks. We consider two different channel state information (CSI) scenarios: 1) no transmit CSI at the base station and receive CSI at the multicast nodes; 2) channel knowledge at the base station of base-station-to-users channels but not of intra-user channels, and receive CSI at the multicast nodes. Results show that cooperation is worthwhile for sufficiently large networks and for the low SNR regime.
\end{abstract}

\section{INTRODUCTION}

Wireless multicast networks consist of a single source node (i.e., the base station) transmitting simultaneously a unique message to $N$ destination nodes (i.e., the multicast nodes), e.g., for radio and television broadcasting. Towards this goal, the source node exploits the broadcast nature of wireless channels, and, in order to serve simultaneously all the users, it typically adjusts its transmission rate to the user suffering the worst channel conditions. Hence, one-hop multicasting is severely affected by multipath fading. Indeed, for $N \rightarrow \infty$, the reliable transmission rate of one-hop multicasting, under independently faded channels, converges almost surely to zero [1]. One approach to solve such reliability problem is cooperative diversity [2].

Cooperative diversity has been widely studied as a means to combat fading in wireless networks. In cooperative systems, network users relay each other's messages in order to obtain diversity and increase capacity by combining the received signal at the physical layer [3] [4]. The basic model for cooperation is the single relay channel and it was studied by Cover and El Gamal in [5]. Rate gains are obtained therein by using regenerative (decode-and-forward and partial decoding) and non regenerative (compress-and-forward) coding schemes for relaying, assuming a single source-destination pair. The extension of cooperative techniques to the multiplesource single-destination scenario was carried out in [6], where orthogonal repetition and space-time coding relaying schemes were shown to yield full spatial diversity gains. As an extension, physical layer multi-hopping was first presented in [4], again considering one destination node and multiple

This work was partially funded by the Generalitat de Catalunya under grant SGR-2005-00690 and by the European Comission under project IST-2002507525

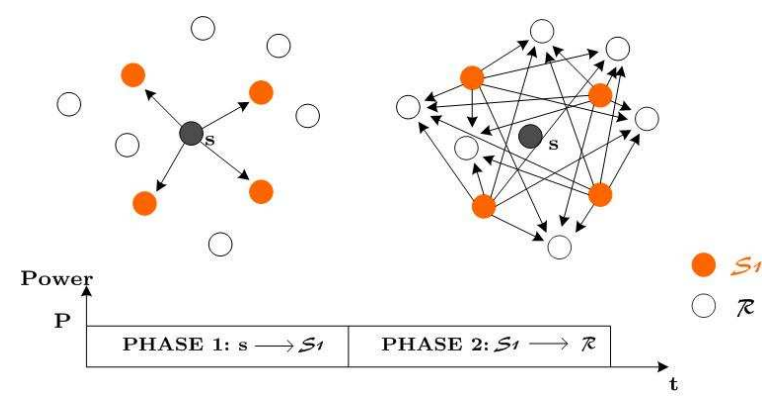

Fig. 1. Space-Time coded cooperative multicasting

sources conferencing. The aim of this paper is to generalize these results to the single-source multiple-destination scenario, i.e., to cooperative multicasting [7].

The fundamental capacity limit on cooperative multicasting has been recently obtained in [8]. In the limit of a large number of multicast users, cooperation is shown to effectively overcome fading, making the reliable multicast transmission rate $^{1}$ equal to $[8$, Theorem 1$]$ :

$$
\mathcal{C}=\log _{2}\left(1+\frac{\mathrm{P}}{\sigma_{o}^{2}}\right),
$$

with $\mathrm{P}$ being the total transmitted power, $\sigma_{o}^{2}$ the noise power, and assuming zero mean, unitary-power, Rayleigh-faded channels among network nodes.

In this paper, we analyze the performance of a two-phase, space-time coded, cooperative multicast protocol in finite size networks, and compare it with the limit (1). According to the protocol, the source node uses the first phase to transmit the message to the subset of users with best channel to the source, who decode it. Then, in a second phase, these users reencode and jointly relay data to the remaining set of users by using distributed space-time codes [9] (see Fig 1). We analyze separately two channel state information (CSI) scenarios. 1) No transmit CSI neither at the source nor at the relay nodes, but receive channel knowledge at the multicast nodes. This scenario models networks without base stationto-users feedback. 2) Broadcast CSI at the base station (i.e., updated knowledge of its channel to the multicast users) but no knowledge of user-to-user channels. At the multicast nodes, we assume only receive CSI. This scenario models cellular wireless networks with channel feedback and allows the base station to optimize resource allocation.

Throughout the paper, we use the outage capacity as the performance metric and derive the latter for the two CSI

\footnotetext{
${ }^{1}$ Rate decoded by all multicast users with arbitrarily small error probability.
} 
scenarios under study. Results are compared with the outage capacity of a single hop non-cooperative multicasting. The remainder of the paper is organized as follows: Section II states the network model and definitions. In Section III, we analyze the non-cooperative multicasting, while the twophase cooperative protocol is studied in Section IV. Finally, numerical results are presented in Section V.

\section{Network Model AND DEFinitions}

We consider a wireless multicast network with a source node $s$ (the base station) and a set of receiver nodes $\mathcal{N}=$ $\{1,2, \cdots, N\}$ (the multicast users). Aiming at transmitting data to users, a cooperative multicast protocol based upon two concatenated, identical phases, is established (see Fig. 1) [8]:

- Phase 1: the first phase of the protocol is used by the source node to broadcast data. However, considering independently faded source-to-users channels, only a subset $\mathcal{S}_{1} \subset \mathcal{N}$ of users is able to reliably decode data during this phase (henceforth, this subset will be referred to as decoding set of phase 1).

- Phase 2: next, during the second phase, the set of nodes $\mathcal{S}_{1}$ relay the multicast data, using a distributed spacetime code (DSTC) and, thus, create spatial diversity [10]. Relaying consists of the transmission of a copy of the original message mapped over a DSTC. The receiving nodes in phase 2 , i.e., $\mathcal{R}=\mathcal{N}-\mathcal{S}_{1}$, attempt to decode by using the received signal during the second phase. As previously, only a subset of users $\mathcal{S}_{2}$ will be able to decode, and the outage event (denoted by $\mathcal{O}$ ) occurs whenever $S_{1} \cup S_{2} \neq \mathcal{N}$.

To implement this transmission strategy we use the following coding scheme: the source encodes information messages into an independently, identically distributed (i.i.d.) Gaussian codebook $\mathcal{X}_{s} \in \mathrm{C}^{\frac{\mathrm{n}}{2}}$, with $\mathrm{n}$ the total number of transmitted symbols during the two phases. It then selects the message $\omega \in\left[1,2^{\mathrm{nR}}\right]$ for transmission in Phase 1 (with $\mathrm{R}$ the multicast rate) and encodes it using the encoding function $x_{s}:\left\{1, \cdots, 2^{\mathrm{nR}}\right\} \rightarrow \mathcal{X}_{s}$. All network nodes attempt to decode, but just a subset $\mathcal{S}_{1}$ is able to do so, being:

$$
\mathcal{S}_{1}=\left\{i \in \mathcal{N}: \frac{1}{2} \mathcal{I}\left(X_{s} ; Y_{i 1}\right) \geq \mathrm{R}\right\},
$$

$\mathcal{I}(\cdot, \cdot)$ denotes mutual information, and $X_{s}$ is the signal transmitted by the source. Factor $\frac{1}{2}$ follows from the timedivision nature of the protocol, and $Y_{i 1}$ is the received signal at node $i$ during phase 1 . Once decoded $x_{s}(\omega)$, nodes in $\mathcal{S}_{1}$ generate the linear DSTC codeword $\mathbf{x}_{\text {DSTC }}(\omega)$ using a linear transformation $\mathcal{L}: \mathrm{C}^{\frac{n}{2}} \rightarrow \mathrm{C}^{\left|\mathcal{S}_{1}\right| \times \frac{n}{2}}$ on $x_{s}(\omega)$ [11, Chapter 7]. The codeword ${ }^{2}$ is then jointly sent during phase 2 . Finally, users in $\mathcal{R}$ attempt to decode in two steps: first they apply an inverse linear transformation to its received signal during phase 2 in order to decode the DSTC codeword; then, they attempt to decode $x_{s}(\omega)$. Thus, node $i \in \mathcal{R}$ will be able to decode data if and only if:

$$
\mathrm{R} \leq \frac{1}{2} \mathcal{I}\left(\mathbf{X}_{\mathcal{S}_{1}} ; Y_{i 2}\right)
$$

where $\mathbf{X}_{\mathcal{S}_{1}}=\left[X_{1}, \cdots, X_{\left|\mathcal{S}_{1}\right|}\right]^{T}$ is the space-time vector transmitted by the nodes in $\mathcal{S}_{1}$ and the $Y_{i 2}$ the received signal at node $i$ during phase 2 .

\footnotetext{
${ }^{2}$ DSTC design is beyond of the scope of the paper. We address the interested reader to [9].
}

In the protocol, we constrain the per-phase transmitted power to $\mathrm{P}$. However, during phase 2 , the power constraint takes different forms depending on the amount of channel state information. As previously pointed out, our analysis considers two CSI cases: 2) No transmit CSI at the base station, and 2) Broadcast CSI at the base station.

1) For the no transmit CSI case, $\mathcal{S}_{1}$ is unknown and thus random at the transmitted node, as discussed in [6]. Therefore, an instantaneous power constraint can not be enforced. In contrast, an average power constraint is guaranteed by setting ${ }^{3} \mathrm{P}_{n}=\frac{\mathrm{P}}{\mathrm{E}\left\{\left|\mathcal{S}_{1}\right|\right\}}$ for $n \in \mathcal{S}_{1}$, and thus $\mathrm{E}\left\{\sum_{n \in \mathcal{S}_{1}} \mathrm{P}_{n}\right\}=\mathrm{P}$.

2) For the latter, $\mathcal{S}_{1}$ is known at the source node, and then an instantaneous power constraint during phase 2 is enforced by setting the power transmitted by $n \in \mathcal{S}_{1}$ to $\mathrm{P}_{n}=\frac{\mathrm{P}}{\left|\mathcal{S}_{1}\right|}$, where $\left|\mathcal{S}_{1}\right|$ is the cardinality of set $\mathcal{S}_{1}$.

The signal received at the multicast users during the two phases is written as:

$$
\begin{aligned}
& Y_{i 1}=\mathrm{a}_{s, i} \cdot X_{s}+Z_{i}, i \in \mathcal{N} \\
& Y_{i 2}=\sum_{n \in \mathcal{S}_{1}} \mathrm{a}_{n, i} \cdot X_{n}+Z_{i}, i \in \mathcal{R}
\end{aligned}
$$

where $\mathrm{a}_{s, i} \sim \mathcal{C N}(0,1)$ and $\mathrm{a}_{n, i} \sim \mathcal{C N}(0,1)$ are the fading coefficients between the base station and receiver $i$, and node $n$ to receiver $i$, respectively. These are assumed independently distributed and invariant during the entire frame. The term $Z_{i} \sim \mathcal{C N}(0,1)$ is the AWGN at the $i^{\text {th }}$ receiver, with normalized power $\sigma_{o}^{2}=1$. Finally, $X_{s} \sim \mathcal{C N}(0, \mathrm{P})$ is the complex Gaussian codeword transmitted by the source and $\mathbf{X}_{\mathcal{S}_{1}}=\left[X_{1}, \cdots, X_{\left|\mathcal{S}_{1}\right|}\right] \sim \mathcal{C N}\left(0, \frac{\mathrm{P}}{\alpha} \cdot \mathbf{I}\right)$ the complex Gaussian DSTC transmitted by $\mathcal{S}_{1}$ during phase 2 . According to the discussion above, the normalization constant reads $\alpha=\left|\mathcal{S}_{1}\right|$ for the broadcast CSI and $\alpha=\mathrm{E}\left\{\left|\mathcal{S}_{1}\right|\right\}$ for the no CSI case.

According to (4), the mutual information at the receiver nodes at the end of phase 1 and 2 respectively is computed as:

$$
\begin{aligned}
& \mathcal{I}_{i 1}=\frac{1}{2} \log _{2}\left(1+\left|\mathrm{a}_{s, i}\right|^{2} \mathrm{P}\right), i \in \mathcal{N} \\
& \mathcal{I}_{i 2}=\frac{1}{2} \log _{2}\left(1+\frac{\left|\mathbf{h}_{i 2}\right|^{2}}{\alpha} \cdot \mathrm{P}\right), i \in \mathcal{R}
\end{aligned}
$$

where $\mathbf{h}_{i 2}=\left[\mathrm{a}_{1, i}, \cdots, \mathrm{a}_{\left|\mathcal{S}_{1}\right|, i}\right]$ is the equivalent MISO channel from $\mathcal{S}_{1}$ to receiver $i$.

Throughout the paper, we consider the multicast outage probability and the multicast outage capacity as the performance metric of the network. Both are defined as follows:

Definition 1: The multicast outage probability is the probability that the transmitted message with a given rate $R$ $[\mathrm{bps} / \mathrm{Hz}]$, is not correctly decoded by at least one multicast user at the end of both phases, i.e.,

$$
P_{o}(\mathrm{R})=\operatorname{Pr}\{\mathcal{O} \mid \mathrm{R}\}
$$

Definition 2: The multicast outage capacity at the outage level $p$ is the maximum transmission rate $\mathrm{R}[\mathrm{bps} / \mathrm{Hz}]$ that guarantees a multicast outage probability lower than or equal to $p$, i.e,

$$
\mathcal{C}_{o}(p)=\max \left\{\mathrm{R}: P_{o}(\mathrm{R}) \leq p\right\}
$$

\footnotetext{
${ }^{3} \mathrm{E}\{\cdot\}$ denotes expectation.
} 


\section{One-Hop Non-Cooperative Multicasting}

In non-cooperative multicasting the base station transmits the data simultaneously to all network nodes in one hop, with power $\mathrm{P}$, and without any cooperative relaying among receivers. The outage capacity of the protocol is then computed as follows:

\section{A. No Transmit CSI}

Without transmit CSI at the source, the outage probability of user $i$ is given by:

$$
\begin{aligned}
\mathrm{P}_{o}^{i}(\mathrm{R}) & =\operatorname{Pr}\left\{\log _{2}\left(1+\left|\mathrm{a}_{s, i}\right|^{2} \mathrm{P}\right)<\mathrm{R}\right\} \\
& =1-e^{-\frac{2^{\mathrm{R}}-1}{\mathrm{P}}},
\end{aligned}
$$

where second equality follows from the cumulative distribution function (cdf) of Rayleigh fading $\mathrm{a}_{s, i}$, i.e, $\mathrm{F}_{\left|\mathrm{a}_{s, i}\right|^{2}}(\mathrm{a})=1-$ $e^{-\mathrm{a}}$. Consequently, considering independently faded channels, the multicast outage probability reads:

$$
\begin{aligned}
\mathrm{P}_{o}(\mathrm{R}) & =1-\prod_{i=1}^{N}\left(1-\mathrm{P}_{o}^{i}(\mathrm{R})\right) \\
& =1-\left(e^{-\frac{{ }^{\mathrm{R}}-1}{\mathrm{P}}}\right)^{N} .
\end{aligned}
$$

Therefore, the multicast outage capacity of one-hop multicasting network, for a given outage level $p$, is derived from Definition 2 as:

$$
\mathcal{C}_{o}(p)=\log _{2}\left(1+\frac{1}{N} \log \left(\frac{1}{1-p}\right) \mathrm{P}\right) .
$$

It is shown that as the number of multicast users $N$ increases, the outage capacity decreases.

\section{B. Broadcast CSI}

With broadcast CSI available at the source (e.g. obtained via feedback), the base station can adapt its transmission rate to the wireless channel conditions. Indeed, it can serve the $N$ network users by adjusting the transmission rate to the user with worst channel conditions. However, for $N \rightarrow \infty$ the achievable rate of this scheme converges almost surely to zero [1]. In order to overcome this limitation, the base station transmits data only to the fraction $1-p$ of users with better channel conditions, while the other fraction $p$ remains in outage. For this case the outage capacity is computed as

$$
\mathcal{C}_{o}(p)=\mathrm{E}\left\{\log _{2}\left(1+\left|\mathrm{a}_{s, p \cdot N}\right|^{2} \cdot \mathrm{P}\right)\right\}
$$

where $\left|\mathrm{a}_{s, p \cdot N}\right|^{2}$ is the $\lfloor p \cdot N\rfloor$-th source-users channel, in a scale from the smallest to the largest.

\section{Two-Phase Space-Time Coded Cooperative Multicasting}

We analyze now the outage capacity and the outage probability of the cooperative multicast protocol presented in Section II. As in the previous secton, both CSI cases are analyzed separately.

\section{A. No Transmit CSI}

Without transmit CSI at the base station, the decoding set during phase 1, i.e., $\mathcal{S}_{1}$, is unknown and random at the source. Hence, the multicast outage probability, for a given transmission rate $\mathrm{R}[\mathrm{bps} / \mathrm{Hz}]$, is given by:

$$
\mathrm{P}_{o}(\mathrm{R})=\sum_{\mathcal{S}_{1}} \operatorname{Pr}\left\{\mathcal{S}_{1}\right\} \cdot \operatorname{Pr}\left\{\mathcal{O} \mid \mathrm{R}, \mathcal{S}_{1}\right\}
$$

where $\operatorname{Pr}\left\{\mathcal{S}_{1}\right\}$ is the probability of a given decoding set $\mathcal{S}_{1}$ during phase 1 to occur, and $\operatorname{Pr}\left\{\mathcal{O} \mid \mathrm{R}, \mathcal{S}_{1}\right\}$ the multicast outage probability at phase 2 , given such decoding set at phase 1 . The former can be computed from the mutual information in (5), and noting that fading channels are independent, identically distributed (i.i.d.), as:

$$
\operatorname{Pr}\left\{\mathcal{S}_{1}\right\}=\prod_{i \in \mathcal{S}_{1}} \operatorname{Pr}\left\{\mathcal{I}_{i 1} \geq \mathrm{R}\right\} \prod_{i \notin \mathcal{S}_{1}} \operatorname{Pr}\left\{\mathcal{I}_{i 1}<\mathrm{R}\right\} .
$$

Furthermore, recalling that $\left|\mathrm{a}_{s, i}\right|^{2}$ is a unitary-mean exponential random variable with $\operatorname{Pr}\left\{\mathcal{I}_{i 1} \geq \mathrm{R}\right\}=e^{-\frac{2^{2 \mathrm{R}}-1}{\mathrm{P}}}$. Then, we rewrite (14) as:

$$
\operatorname{Pr}\left\{\mathcal{S}_{1}\right\}=\left(e^{-\frac{2^{2 \mathrm{R}-1}}{\mathrm{P}}}\right)^{\left|\mathcal{S}_{1}\right|}\left(1-e^{-\frac{2^{2 \mathrm{R}-1}}{\mathrm{P}}}\right)^{N-\left|\mathcal{S}_{1}\right|} .
$$

On the other hand, $\operatorname{Pr}\left\{\mathcal{O} \mid \mathrm{R}, \mathcal{S}_{1}\right\}$ defines the probability that, given the decoding set $\mathcal{S}_{1}$ during phase 1 , there exists at least one node belonging to $\mathcal{N}-\mathcal{S}_{1}$ during phase 2 that is not able to decode the space-time coded signal. Recalling that we have i.i.d. channels in the network, this probability is computed from the probability of outage of every single receiver of phase 2:

$$
\begin{aligned}
\operatorname{Pr}\left\{\mathcal{O} \mid \mathrm{R}, \mathcal{S}_{1}\right\} & =1-\prod_{i \notin \mathcal{S}_{1}}\left(1-\operatorname{Pr}\left\{\mathcal{I}_{i 2}<\mathrm{R}\right\}\right) \\
= & 1-\prod_{i \notin \mathcal{S}_{1}}\left(1-\operatorname{Pr}\left\{\frac{\left|\mathbf{h}_{i 2}\right|^{2}}{\alpha}<\frac{2^{2 \mathrm{R}}-1}{\mathrm{P}}\right\}\right),
\end{aligned}
$$

with $\alpha=\mathrm{E}\left\{\left|\mathcal{S}_{1}\right|\right\}$ to satisfy the power constraint. To evaluate the unicast outage probability, we first notice that $\left|\mathbf{h}_{i 2}\right|^{2}=$ $\sum_{j \in \mathcal{S}_{1}}\left|\mathrm{a}_{j, i}\right|^{2}$ is a chi-squared distributed random variable with $2 \cdot\left|\mathcal{S}_{1}\right|$ degrees of freedom, i.e. $\left|\mathbf{h}_{i 2}\right|^{2} \sim \mathcal{X}_{2 \cdot\left|\mathcal{S}_{1}\right|}^{2}$. Likewise, the cdf of $\mathcal{X}_{2 \cdot\left|\mathcal{S}_{1}\right|}^{2}$ is the regularized incomplete gamma function, $\mathrm{F}_{\mathcal{X}_{2 \cdot\left|\mathcal{S}_{1}\right|}^{2}}(b)=\gamma\left(\left|\mathcal{S}_{1}\right|, b\right)=1 /\left(\left|\mathcal{S}_{1}\right|-1\right) ! \cdot \int_{o}^{b} \mathrm{x}^{\left|\mathcal{S}_{1}\right|-1} e^{-\mathrm{x}} d \mathrm{x}$. Hence, we evaluate

$$
\operatorname{Pr}\left\{\frac{\left|\mathbf{h}_{i 2}\right|^{2}}{\alpha}<\frac{2^{2 \mathrm{R}}-1}{\mathrm{P}}\right\}=\gamma\left(\left|\mathcal{S}_{1}\right|, \alpha \cdot \frac{2^{2 \mathrm{R}}-1}{\mathrm{P}}\right) .
$$

Therefore, plugging (17) with $\alpha=\mathrm{E}\left\{\left|\mathcal{S}_{1}\right|\right\}$ into (16), and both in (13) with (15), we obtain the outage probability in (18) (See next page on the top). Notice that, in order to derive the combinatorial in (18), we use the fact that for two different decoding sets during phase $1, \mathcal{S}_{1}=\mathcal{A}$ and $\mathcal{S}_{1}=\mathcal{B}$, the probabilities $\operatorname{Pr}\{\mathcal{A}\} \cdot \operatorname{Pr}\{\mathcal{O} \mid \mathrm{R}, \mathcal{A}\}=\operatorname{Pr}\{\mathcal{B}\} \cdot \operatorname{Pr}\{\mathcal{O} \mid \mathrm{R}, \mathcal{B}\}$ if $|\mathcal{A}|=|\mathcal{B}|$. Finally, the outage capacity of the proposed cooperative multicasting is obtained by inverting equation (18) following Definition 2. 


$$
P_{o}(\mathrm{R})=\sum_{n=0}^{N}\left(\begin{array}{l}
N \\
n
\end{array}\right)\left(e^{-\frac{2^{2 \mathrm{R}-1}}{\mathrm{P}}}\right)^{n}\left(1-e^{-\frac{2^{2 \mathrm{R}-1}}{\mathrm{P}}}\right)^{N-n}\left(1-\left(1-\gamma\left(n, \mathrm{E}\left\{\left|\mathcal{S}_{1}\right|\right\} \cdot \frac{2^{2 \mathrm{R}}-1}{\mathrm{P}}\right)\right)^{N-n}\right)
$$

$$
\mathcal{C}_{o}(p)=\max _{1 \leq \kappa \leq N-1} \min \left\{\frac{1}{2} \log _{2}\left(1+\left|\mathrm{a}_{s, \kappa}\right|^{2} \mathrm{P}\right), \frac{1}{2} \log _{2}\left(1+\gamma^{-1}\left(\kappa, 1-(1-p)^{\frac{1}{N-\kappa}}\right) \cdot \frac{\mathrm{P}}{\kappa}\right)\right\}
$$

\section{B. Broadcast CSI}

We analyze now a multicast network where the source node has source-to-users transmit channel knowledge, but is unaware (as the nodes in the decoding set $\mathcal{S}_{1}$ ) of the intrauser channel coefficients. As for one-hop multicasting, the base station utilizes the channel knowledge to adapt its transmission rate to the channel conditions. The optimal approach prescribes the selection of the decoding set $\mathcal{S}_{1}$ during phase 1 , so that the outage probability during phase 2 is minimized. Intuitively, a trade-off arises when selecting $\mathcal{S}_{1}$ : the higher is the cardinality of $\mathcal{S}_{1}$, the larger is the outage capacity of space-time coded transmission. However, in order to increase the cardinality of $\mathcal{S}_{1}$, the base station has to reduce its transmission rate so more nodes can decode during the first phase. The source must trade between both effects, as shown in Proposition 1.

First, let us consider without loss of generality that the base station orders the multicast users according to:

$$
\left|\mathrm{a}_{s, 1}\right| \geq \ldots \geq\left|\mathrm{a}_{s, i}\right| \geq \ldots \geq\left|\mathrm{a}_{s, N}\right|,
$$

and define $\mathcal{S}_{1}^{\kappa}$ as the set of nodes $[1, \cdots, \kappa]$.

Proposition 1: The outage capacity of a two-phase, spacetime coded, cooperative multicasting with broadcast CSI is given by

$$
\mathcal{C}_{o}(p)=\max _{1 \leq \kappa \leq N-1} \min \left\{\mathcal{C}_{1}^{\kappa}, \mathcal{C}_{2}^{\kappa}(p)\right\}
$$

where

$$
\begin{aligned}
& \mathcal{C}_{1}^{\kappa}:=\frac{1}{2} \log _{2}\left(1+\left|\mathrm{a}_{s, \kappa}\right|^{2} \mathrm{P}\right) \\
& \mathcal{C}_{2}^{\kappa}(p):=\max \left\{\mathrm{R}: \operatorname{Pr}\left\{\mathcal{O} \mid \mathrm{R}, \mathcal{S}_{1}^{\kappa}\right\}=p\right\}
\end{aligned}
$$

Remark: When interpreting (21), we remark that $\min \left\{\mathcal{C}_{1}^{\kappa}, \mathcal{C}_{2}^{\kappa}(p)\right\}$ is the outage capacity when the source selects the set $\mathcal{S}_{1}^{\kappa}$ to decode during phase 1 . Due to ordering (20), $\mathcal{C}_{1}^{\kappa}$ is the maximum reliable rate at which the base station communicates with arbitrary small error probability with $\mathcal{S}_{1}^{\kappa}$. Moreover, $\mathcal{C}_{2}^{\kappa}(p)$ is the maximum transmission rate during phase 2 that, given the decoding set $\mathcal{S}_{1}^{\kappa}$, guarantees an outage level lower than or equal to $p$. Hence, the minimum of both determines the outage capacity for $\mathcal{S}_{1}^{\kappa}$, and the minimization over $\kappa$, the optimum decoding set.

Proof: Let us consider a half-duplex multiple relay channel with a single source node, a set of relay nodes $\mathcal{S}_{1}^{\kappa}$, and a set of destination nodes $\mathcal{N}-\mathcal{S}_{1}^{\kappa}$ [4], [12], [13]. For this relay channel, let us assume that relay nodes jointly relay data under a decode-and-forward strategy, and use space-time codes. Furthermore, assume no direct path from source to destinations. It is straightforward to notice that this channel models a two-hop multicasting with the decoding set during phase 1 equal to $\mathcal{S}_{1}^{\kappa}$.

In [13, Proposition 2] the analysis of half-duplex decodeand-forward (D\&F) relay channels was presented. Results therein show that the source-destination achievable rate for D\&F relay channels ${ }^{4}$ equals the minimum of the source-relays

\footnotetext{
${ }^{4}$ With no direct path from source to destination.
}

rate and the relays-destination rate. In the multicast network with $\mathcal{S}_{1}^{\kappa}$ (modelled above as a multiple relay channel), the relays to destination capacity of outage, for a given outage level $p$, is equal to:

$$
\mathcal{C}_{2}^{\kappa}(p)=\max \left\{\mathrm{R}: \operatorname{Pr}\left\{\mathcal{O} \mid \mathrm{R}, \mathcal{S}_{1}^{\kappa}\right\}=p\right\} .
$$

On the other hand, the maximum source to relays transmission rate, reliably decoded by all nodes in $\mathcal{S}_{1}^{\kappa}$, is equal to:

$$
\begin{aligned}
\mathcal{C}_{1}^{\kappa} & =\min _{i \in \mathcal{S}_{1}^{\kappa}} \frac{1}{2} \log _{2}\left(1+\left|\mathrm{a}_{s, i}\right|^{2} \mathrm{P}\right) \\
& =\frac{1}{2} \log _{2}\left(1+\left|a_{s, \kappa}\right|^{2} \mathrm{P}\right),
\end{aligned}
$$

where second equality follows from ordering (20). Therefore, applying the previously mentioned [13, Proposition 2], we obtain that the maximum transmission rate, for a given outage level $p$, and for a given decoding set $\mathcal{S}_{1}^{\kappa}$, is

$$
\mathcal{C}^{\kappa}=\min \left\{\mathcal{C}_{1}^{\kappa}, \mathcal{C}_{2}^{\kappa}(p)\right\}
$$

Finally, the base station select the decoding set of phase 1 $\mathcal{S}_{1}$ out of $\left\{\mathcal{S}_{1}^{1}, \cdots, \mathcal{S}_{1}^{\kappa}, \cdots \mathcal{S}_{1}^{N-1}\right\}$ in order to maximize the outage capacity, which concludes the proof. Notice, that $\mathcal{S}_{1}$ may not be $\mathcal{S}_{1}^{0}$ or $S_{1}^{N}$, since this turns our problem into the non-cooperative multicasting. $\diamond$

To evaluate the outage capacity in (21), we first provide a closed form expression for $\mathcal{C}_{2}^{\kappa}(p)$ in (23). This rate is defined in terms of $\operatorname{Pr}\left\{\mathcal{O} \mid \mathrm{R}, \mathcal{S}_{1}^{\kappa}\right\}$, which is computed as:

$\operatorname{Pr}\left\{\mathcal{O} \mid \mathrm{R}, \mathcal{S}_{1}^{\kappa}\right\}=1-\prod_{i \notin \mathcal{S}_{1}^{\kappa}}\left(1-\operatorname{Pr}\left\{\frac{\left|\mathbf{h}_{i 2}\right|^{2}}{\alpha}<\frac{2^{2 \mathrm{R}}-1}{\mathrm{P}}\right\}\right)$

where, as in (17), we have:

$$
\operatorname{Pr}\left\{\frac{\left|\mathbf{h}_{i 2}\right|^{2}}{\alpha}<\frac{2^{2 \mathrm{R}}-1}{\mathrm{P}}\right\}=\gamma\left(\left|\mathcal{S}_{1}^{\kappa}\right|, \alpha \cdot \frac{2^{2 \mathrm{R}}-1}{\mathrm{P}}\right),
$$

with $\left|\mathcal{S}_{1}^{\kappa}\right|=\kappa$ by definition and $\alpha=\left|\mathcal{S}_{1}^{\kappa}\right|$ to satisfy the power constraint. Hence, plugging (28) into (27) and applying definition (23) we obtain

$\mathcal{C}_{2}^{\kappa}(p)=\frac{1}{2} \log _{2}\left(1+\gamma^{-1}\left(\kappa, 1-(1-p)^{\frac{1}{N-\kappa}}\right) \cdot \frac{\mathrm{P}}{\kappa}\right)$,

with $\mathrm{b}=\gamma^{-1}(\kappa, \mathrm{a})$ the inverse function of the regularized incomplete gamma function, i.e., $\mathrm{a}=\gamma(\kappa, \mathrm{b})$. Therefore, making use of Proposition 1 we obtain the outage capacity as in (19).

\section{Numerical Results}

In this section, we evaluate the outage capacity of the twophase, space-time coded, cooperative protocol and compare it with the outage capacity of non-cooperative multicasting. For convenience of representation, we normalize the outage capacity in all plots with respect to the bound in (1).

Fig. 2 depicts the outage capacity of both protocols versus the total number of multicast users, $N$. We consider a multicast outage level of $p=10^{-1}$ and a transmit signal-to-noise ratio of $10 \mathrm{~dB}$, i.e., $\frac{\mathrm{P}}{\sigma_{o}^{2}}=10 \mathrm{~dB}$. Moreover, for the broadcast 


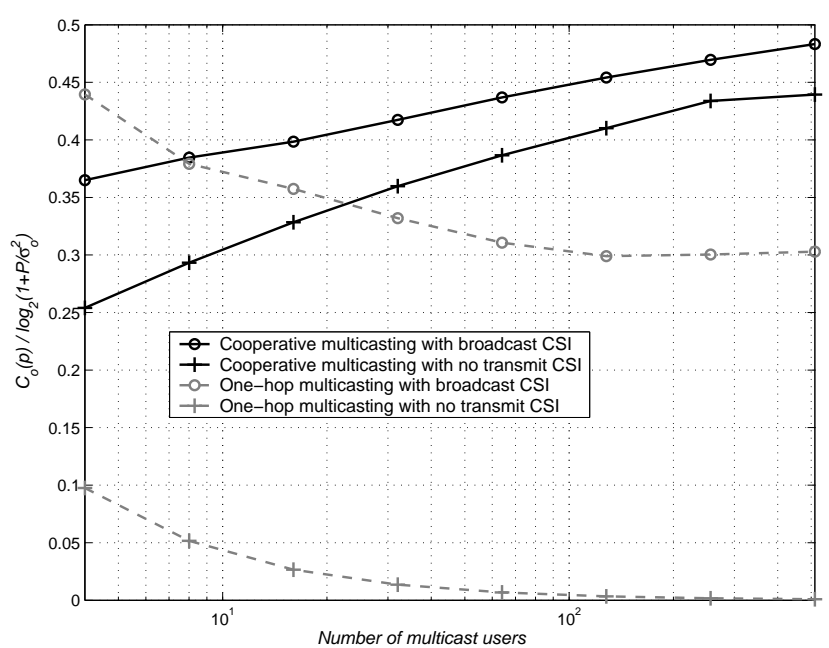

Fig. 2. Outage capacity versus the number of multicast users, for a two phase, space-time coded, cooperative multicasting. The outage capacity of non-cooperative multicasting is also plotted as reference. Moreover, both CSI cases are depicted, and SNR $=10 \mathrm{~dB}$ and $p=10^{-1}$ are assumed.

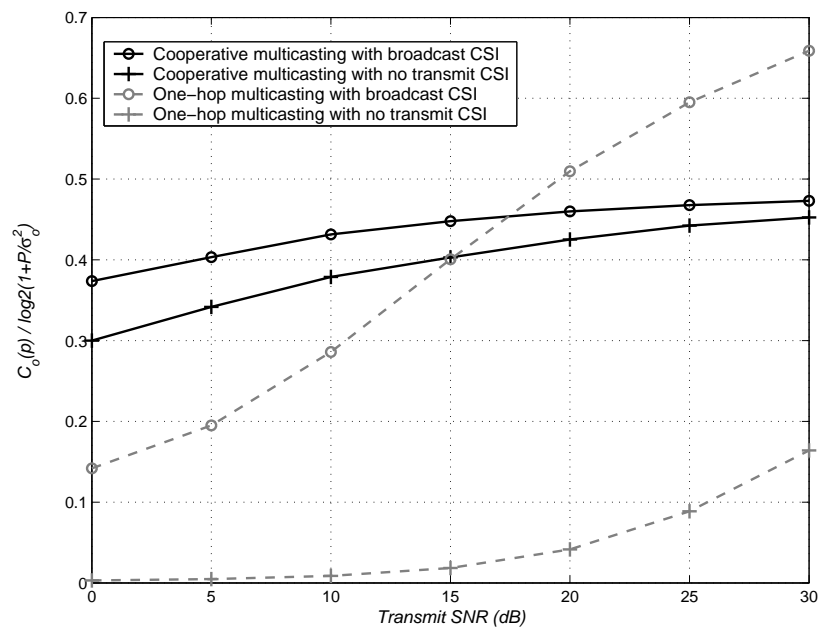

Fig. 3. Outage capacity versus the transmitted SNR for a two phase, space-time coded, cooperative multicasting. The outage capacity of noncooperative multicasting is also plotted as reference. Moreover, both CSI cases are depicted, and 50 multicast users and $p=10^{-1}$ are assumed.

CSI case, we plot the expected outage capacity in (12) and (19), averaged over the channel distribution. Firstly, results show that for both CSI cases, the capacity of the one-hop multicasting protocol decreases when increasing the number of users. On the contrary, the outage capacity of the cooperative protocol increases when increasing the number of users, due to the amount of spatial diversity introduced in the system. In fact, for the no transmit CSI case, cooperation among nodes is advantageous for any $N$. However, for the Broadcast CSI scenario, cooperation is only useful for a number of users large enough (i.e., $\mathbf{N} \geq 10$ ). Finally, providing Broadcast CSI at the base station of a cooperative multicasting is more advantageous with small $N$. Moreover, we notice that the performance of the cooperative protocol is far from the fundamental limit in [8], when the number of nodes is not infinite.

Fig. 3 depicts outage capacity results versus the transmit SNR, considering $p=10^{-1}$ and 50 multicast users. First, we notice that all capacities increase as $\frac{P}{N_{o}}$ increases; however the growth is more significant for the non-cooperative multicast. Second, results show that for the cooperative protocol, the effect of CSI decreases as the SNR increases. Finally, we notice that the cooperative multicasting always outperforms the noncooperative protocol when no transmit CSI is available. On the other hand, for the Broadcast CSI case, cooperation is only worthwhile in the low SNR regime. This can be explained by noticing that the proposed protocol is based upon decode-andforward relaying, which is inefficient in the high SNR regime [14].

\section{Conclusions}

In this paper, a cooperative multicast protocol were proposed in order to overcome multi-path fading in wireless multicasting. The proposed protocol is based on two consecutive, identical phases, and on decode-and-forward relaying. The first phase is used by the base station to broadcast data within the network. Next, during the second phase, the decoding set of the first slot, jointly relay data by using distributed spacetime codes. For the protocol, we analyzed the outage capacity under two CSI scenarios: No transmit CSI at the base station, and Broadcast CSI at the base station. Results showed that: i) for the Mo CSI case, cooperation always outperforms onehop multicasting, with up to threefold gains, $i i$ ) with Broadcast CSI, cooperation only increases the outage capacity in the low SNR regime and with large number of multicast users.

\section{REFERENCES}

[1] N. Jindal and Z.Q. Luo, "Capacity limits of multiple antenna multicast," in Proc. International Symposium on Information Theory, Cambridge, USA, Jul. 2006, pp. 1841-1845.

[2] E. Zimermann, P. Herhold, and G. Fettweis, "On the performance of cooperative relaying protocols in wireless networks," European Trans. on Telecommunications, vol. 16, no. 1, pp. 5-16, Jan. 2005.

[3] D. Tse, "Cooperative communication: diversity, freedom and energy," in MSRI Workshop on Mathematics of Relaying and Cooperation in Communication Networks, Berkeley, CA, USA, Apr. 2006.

[4] G. Kramer, M. Gastpar, and P. Gupta, "Cooperative strategies and capacity theorems for relay networks," IEEE Trans. on Information Theory, vol. 51, no. 9, pp. 3037-3063, Sep. 2005.

[5] T. Cover and A. El Gamal, "Capacity theorems for the relay channel," IEEE Trans. on Information Theory, vol. 25, no. 5, pp. 572-584, Sep. 1979.

[6] J.N. Laneman and G. Wornell, "Distributed space-time coded protocols for exploiting cooperative diversity in wireless networks," IEEE Trans. on Information Theory, vol. 49, no. 10, pp. 2415-2425, Oct. 2003.

[7] I. Maric and R.D. Yates, "Cooperative multicast for maximum network lifetime," IEEE Journal on Selected Areas in Communications, vol. 23 , no. 1, pp. 127-135, Jan 2005.

[8] A. Khisti, U. Erez, and G.W. Wornell, "Fundamental limits and scaling behavior of cooperative multicasting in wireless networks," to appear IEEE Trans. on Information Theory, 2006.

[9] B. Sirkeci-Mergen and A. Scaglione, "Randomized distributed spacetime coding for cooperative communication in self organized networks," in Proc. IEEE $6^{\text {th }}$ Workshop on Signal Processing Advances in Wireless Communications, Philadelphia, USA, Mar. 2005, pp. 500-504.

[10] L. Lampe, R. Schober, and S. Yiu, "Distributed space-time coding for multihop transmission in power line communication networks," IEEE Journal on Selected Areas in Communications, vol. 24, no. 7, pp. 13891400, Jul. 2006.

[11] E.G. Larsson and P. Stoica, Space-Time Block Coding for Wireless Communications, $1^{\text {st }}$ Edition, Cambridge University Press, 2003.

[12] A. del Coso and C. Ibars, "Partial decoding for synchronous and asynchronous Gaussian multiple relay channels," in Proc. International Conference on Communications (ICC), Glasgow, UK, Jun. 2007, submitted.

[13] A. Host-Madsen and J. Zhang, "Capacity bounds and power allocation for wireless relay channels," IEEE Trans. on Information Theory, vol. 51, no. 6, pp. 2020-2040, Jun. 2005.

[14] A. El Gamal, M. Mohseni, and S. Zahedi, "Bounds on capacity and minimum energy-per-bit for AWGN relay channels," IEEE Trans. on Information Theory, vol. 52, no. 4, pp. 1545-1561, Apr. 2006. 